\title{
Technè
}

La science au service de l'histoire de l'art et de la préservation des biens culturels

46 | 2018

Science et conservation

\section{Gustave Courbet : une dérestauration spectaculaire du Cerf dans la forêt}

Gustave Courbet: a spectacular derestoration of Cerf dans la Forêt

Pauline Hélou-de La Grandière, Claire Gerin-Pierre, Bruno Mottin et Hélène Tarantola

\section{(2) OpenEdition}

\section{Journals}

Édition électronique

URL : http://journals.openedition.org/techne/353

DOI : 10.4000/techne.353

ISSN : 2534-5168

Éditeur

C2RMF

Édition imprimée

Date de publication : 1 décembre 2018

Pagination : $39-43$

ISBN : 978-2-11-152829-1

ISSN : 1254-7867

Référence électronique

Pauline Hélou-de La Grandière, Claire Gerin-Pierre, Bruno Mottin et Hélène Tarantola, « Gustave Courbet : une dérestauration spectaculaire du Cerf dans la forêt », Technè [En ligne], 46 | 2018, mis en ligne le 19 décembre 2019, consulté le 24 juillet 2020. URL : http://journals.openedition.org/techne/ 353 ; DOI : https://doi.org/10.4000/techne.353

\section{(c) $(1) \ominus$}

La revue Technè. La science au service de l'histoire de l'art et de la préservation des biens culturels est mise à disposition selon les termes de la Licence Creative Commons Attribution - Pas d'Utilisation Commerciale - Pas de Modification 4.0 International. 


\section{Pauline Hélou-de La Grandière Gustave Courbet : une dérestauration Claire Gerin-Pierre \\ Bruno Mottin

spectaculaire du Cerf dans la forêt

Hélène Tarantola

Gustave Courbet: a spectacular derestoration

of Cerf dans la Forêt

Résumé. À l'occasion de la réouverture après rénovation du musée du château de Flers (Orne), Le Cerf dans la forêt de Gustave Courbet (1819-1877) a été confié au C2RMF pour sa restauration. L'œuvre, datée de 1867, présentait un aspect peu habituel lié à des restaurations antérieures très abusives qui avaient provoqué des doutes concernant son attribution : un rentoilage en avait modifié le format initial, l'ensemble avait été défiguré par des repeints masquant l'original sur près de $75 \%$ de la surface et par un vernis "à craqueler ", épais et oxydé, qui provoquait des soulèvements de la couche picturale. La restauration a permis de retrouver une lisibilité de la surface picturale digne d'une æuvre de Courbet.

Mots-clés. Restauration, dérestauration, vernis « $\grave{a}$ craqueler", nettoyage, Gustave Courbet, Flers, musée du château.

\begin{abstract}
Coinciding with the reopening of the Musée du Château de Flers (Orne) after its renovation, Le Cerf dans la Forêt (Deer in the Forest) by Gustave Courbet (1819-77) was entrusted to the C2RMF for restoration. Dating from 1867, the painting had a very unusual appearance due to improper earlier restorations that had raised doubts about its attribution: the initial format had been modified when the canvas was remounted; the entire work had been disfigured by inpainting that concealed nearly $75 \%$ of the surface and by thick oxidized crackle varnish, which caused lifting in the paint layer. The restoration enabled us to reestablish the legibility of a painting worthy of a work by Courbet.
\end{abstract}

Keywords. Restoration, derestoration, crackle varnish, cleaning, Gustave Courbet, Musée du Château de Flers.
Le Cerf dans la forêt (fig. 1) (inv. 1919.1.217), signé et daté « Courbet 67 », est entré dans les collections du musée du château de Flers avec le legs de la collection Julien Salles (philanthrope et ancien maire de Flers), en 19191. Avant son entrée dans les collections, l'œuvre avait fait l'objet d'interventions de restauration qui n'ont pas été documentées : il s'agit d'un rentoilage à la cire, de repeints grossiers couvrant près des trois quarts de la surface et d'un vernis craquelé artificiellement nappant l'ensemble. Une restauration était donc nécessaire pour se rapprocher de l'aspect initial de l'œuvre, en la débarrassant de ces interventions intrusives et maladroites.

\section{L'œuvre}

Le tableau est inconnu des principaux biographes de Courbet, notamment de Robert Fernier et Pierre Courthion ; il n’a jamais été exposé ailleurs qu'au musée de Flers. La négligence dont il est victime pourrait être due à l'importance des restaurations qu'il a subies autrefois ; celles-ci sont si intrusives que la compréhension des éléments constitutifs du tableau a demandé une étude préliminaire au laboratoire (notamment des examens radiographiques) avant de pouvoir envisager la reprise de restauration.

Au revers, la toile originale était non seulement rentoilée, comme cela est fréquent, mais également noyée sous une couche de cire grossièrement étendue (fig. 2). En bordure de toile, la présence d'une toile de gaze ( $8 \mathrm{fils} / \mathrm{cm}$ ), qui a fortement marqué la couche picturale, a laissé supposer que l'œuvre pouvait avoir été transposée de toile sur toile. Cette hypothèse a été infirmée grâce à l'étude de la radiographie : la toile de gaze était visible directement sous la couche picturale en périphérie, à cause d'agrandissements sur tous les bords $(2,5 \mathrm{~cm}$ en hauteur et $3,5 \mathrm{~cm}$ en longueur), modifiant le format original de 72 x $93 \mathrm{~cm}$ à 75 x $100 \mathrm{~cm}$. La présence de guirlandes de tension en périphérie de l'œuvre a bien confirmé que la composition originale n’avait pas été coupée. Cette toile originale est d'armure simple, d'une contexture d'environ 11 x 12 fils au $\mathrm{cm}^{2}$. Le format initial de $72 \times 93 \mathrm{~cm}$ est un format de standard n 30 , fréquemment utilisé jusqu'au XIX ${ }^{\mathrm{e}}$ siècle $^{2}$.

Pauline Hélou-de La Grandière, restauratrice du patrimoine indépendante, spécialité peintures (lagrandiere@gmail.com). Claire Gerin-Pierre, conservateur du patrimoine, département Restauration, C2RMF (claire.gerin-pierre@culture.gouv.fr). Bruno Mottin, conservateur du patrimoine, département Recherche, C2RMF (bruno.mottin@culture.gouv.fr). Hélène Tarantola, responsable du service patrimoine culturel, musée du château de Flers (htarantola@flers-agglo.fr). 


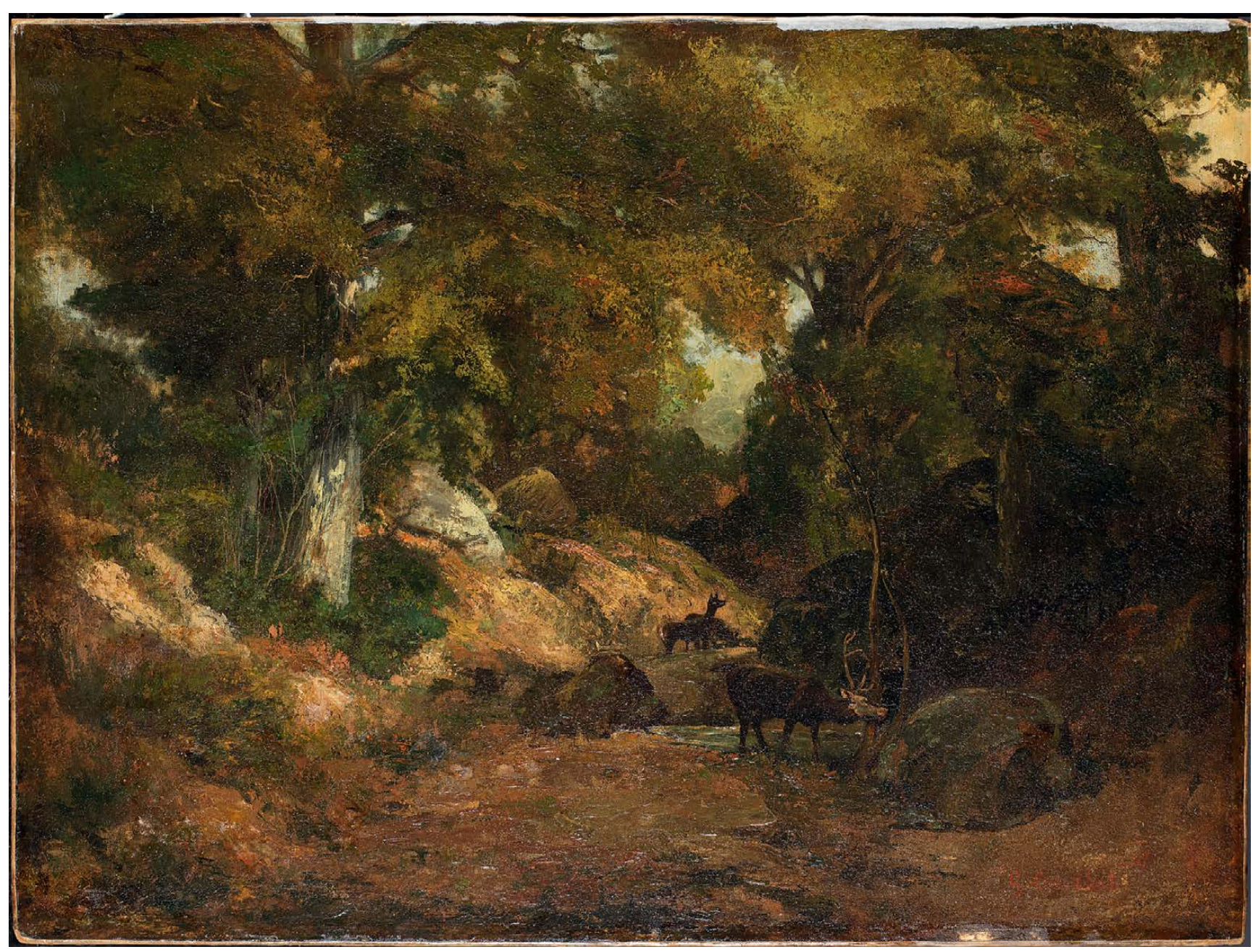

Fig. 1. Gustave Courbet, Le Cerf dans la forêt, 1867, huile sur toile $(75 \times 100 \mathrm{~cm})$, musée du château de Flers, vue d'ensemble avant restauration. (C) C2RMF/G. de Puniet.

La toile est tendue sur un châssis à clés équipé de deux montants verticaux, au modèle peu conventionnel (fig. 2), vraisemblablement posé lors de la restauration. Il a remplacé le châssis original qui possédait un montant central unique (observation des marques de châssis sur le réseau de craquelure, très lisible en radiographie) (fig. 3). L'enduit cireux a été étendu au revers après la dépose de la toile de son châssis et avant son remontage. Il semble avoir été posé pour limiter les mouvements de la toile, très réactive, qui était affectée par un trou et de petites amorces de déchirures visibles en radiographie (lacune de toile en bas à dextre de $45 \mathrm{~cm}^{2}$ ). La présence d'un réseau de craquelure directionnel vertical laisse supposer que la toile a été roulée pendant une assez longue durée et conservée dans de mauvaises conditions avant le rentoilage et la remise sur châssis. Le changement de format n'a pas de justification particulière quant à la composition ; il a peut-être été fait pour utiliser un châssis plus solide (sections plus épaisses, assemblages mobiles) ou pour être placé dans un cadre de belle qualité3.
La compréhension de la couche picturale a demandé une attention particulière, tant les restaurations y sont intrusives. La préparation beige est homogène et couvre l'ensemble de la surface, comme l'a confirmé la restauration complète de l'œuvre qui a remis en évidence la présence de nombreuses réserves de préparation. Peu épaisse, celle-ci laisse parfois à découvert la trame de toile originale, particularité qui a été amplifiée par l'opération de rentoilage, qui a écrasé la matière.

La couche picturale était recouverte de repeints très débordants qui transformaient la composition en ajoutant un rocher dans le sous-bois, en modifiant la tonalité du ciel par des aplats grisâtres et des feuillages fantaisistes et en masquant le sol avec d'étranges troncs d'arbres, recouverts d'aplats bruns au premier plan ; tous ces ajouts ont dissimulé à la fois les usures et les réserves. Les tests de solubilité ont montré que ces repeints étaient huileux ; ils ont été posés à la brosse et au couteau. Le vernis contribuait à achever de dénaturer l'aspect du tableau : nappant entièrement la surface, cette résine synthétique de mauvaise qualité, très oxydée, présentait la particularité de créer un réseau de craquelures 


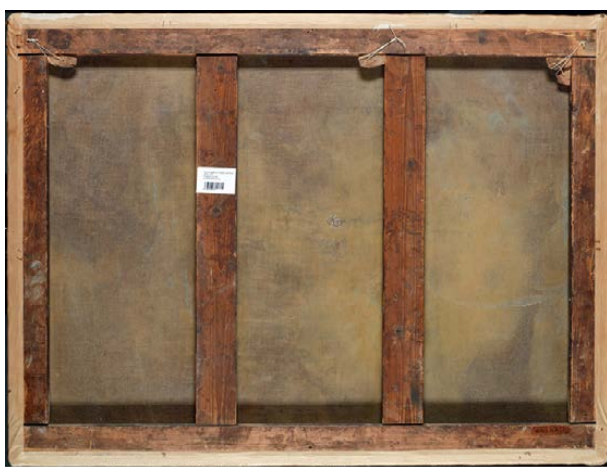

Fig. 2. Gustave Courbet, Le Cerf dans la forêt, 1867, huile sur toile $(75 \times 100 \mathrm{~cm})$, musée du château de Flers, vue d'ensemble du revers. (C) C2RMF/ G. de Puniet.

Fig. 3. Gustave Courbet, Le Cerf dans la forêt, 1867, huile sur toile $(75 \times 100 \mathrm{~cm})$, musée du château de Flers, radiographie. (C) C2RMF/B. Mottin et T. Clot.

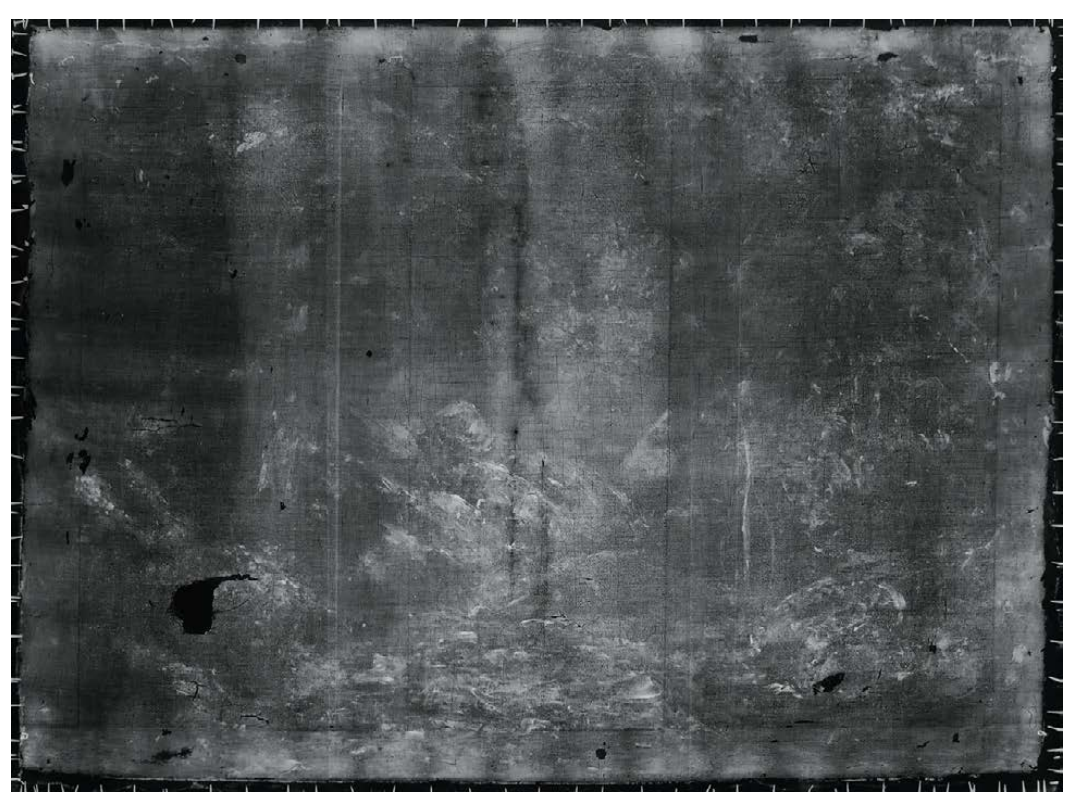

artificielles dans son épaisseur. Ce type de vernis est toujours disponible dans le commerce sous la dénomination «vernis à craqueler ", « vernis craqueleur " ou "vernis à vieillir ». Il s'agit de résines variables devant être appliquées successivement, dont l'une des couches possède la capacité de réticuler avant le séchage de la couche sous-jacente, en créant ainsi un aspect faussement vieilli. Les contraintes de séchage de ce type de vernis sont élevées et en excluent habituellement l'usage en conservation-restauration. Il confère aux œuvres un aspect faussement craquelé qui est généralement l'apanage des faussaires ou des reproductions de faible qualité, et dont l'emploi est dommageable pour un authentique Courbet. Il a sans doute été posé par un restaurateur aux pratiques non muséales pour masquer les usures et homogénéiser les repeints.

La plupart des altérations de l'œuvre sont dues à ces interventions inadaptées. L'altération la plus évolutive et structurelle consiste en des micro-soulèvements répartis sur l'œuvre qui correspondent au réseau de craquelure du vernis : la fine couche picturale se déplaque sous l'effet de la tension engendrée par ce vernis « craqueleur» trop épais.

\section{Restauration}

Les interventions de restauration ${ }^{4}$ ont été réalisées en trois étapes : d'abord la stabilisation des amorces de micro-soulèvements, puis le dévernissage et la suppression des repeints, enfin la réintégration et un nouveau vernissage.

Le refixage a été conduit dans les réserves provisoires du musée, en mars 2014. Ce refixage singulier d'une couche picturale se déplaquant sous l'effet d'un vernis a nécessité la solubilisation partielle de la résine pour relaxer le vernis et replacer ainsi les micro-soulèvements en tête d'épingle. Après avoir testé la solubilité du vernis ${ }^{5}$, plusieurs solvants aux vitesses d'évaporation et de rétention variables ont été testés. L'adhésif choisi pour le refixage dépendait de ce solvant ; il a donc été conduit avec un polymère acrylique en solution Plexigum $\mathrm{P} 675^{6}$ à $4 \%$ dans l'acétate d'éthyle. Après pose d'une goutte de solution au pinceau, l'évaporation a été bloquée avec un film non adhérent $\left(\right.$ Melinex $\left.^{\circledR}\right)$. La remise dans le plan a été faite par séchage de l'adhésif, et maintien de la zone sous poids jusqu'à séchage complet. La méthode employée a permis d'éviter un apport de chaleur qui était trop difficile à maîtriser, compte tenu de l'épaisseur du vernis (fig. 4).

Le rentoilage paraissant stable, il n’a pas été envisagé de le reprendre et la restauration s'est concentrée alors sur la couche picturale.

Le nettoyage a ensuite été réalisé dans les ateliers du C2RMF à Versailles, après réalisation du dossier d'imagerie scientifique. Les tests de solubilité déjà menés ont permis de mettre au point un mélange de solvants adapté à une solubilisation complète du vernis et des repeints. Ce mélange, qui correspond à une alternative à l'acide acétique mis au point par Paolo Cremonesi ${ }^{7}$, est composé d'éthanol $77 \%$, de n-buthyl-acétate $12 \%$ et d'acétone $11 \%$. La suppression de tous les éléments ajoutés, vernis, repeints débordants et mastics débordants est possible avec ce mélange de solvant et par voie mécanique; pour éviter un risque d'usures de la couche picturale qu'auraient engendré de trop nombreux frottements (la couche picturale est potentiellement très épidermée sous les repeints), il a été choisi de tester une alternative aux solvants liquides. Les gels de solvant traditionnels 

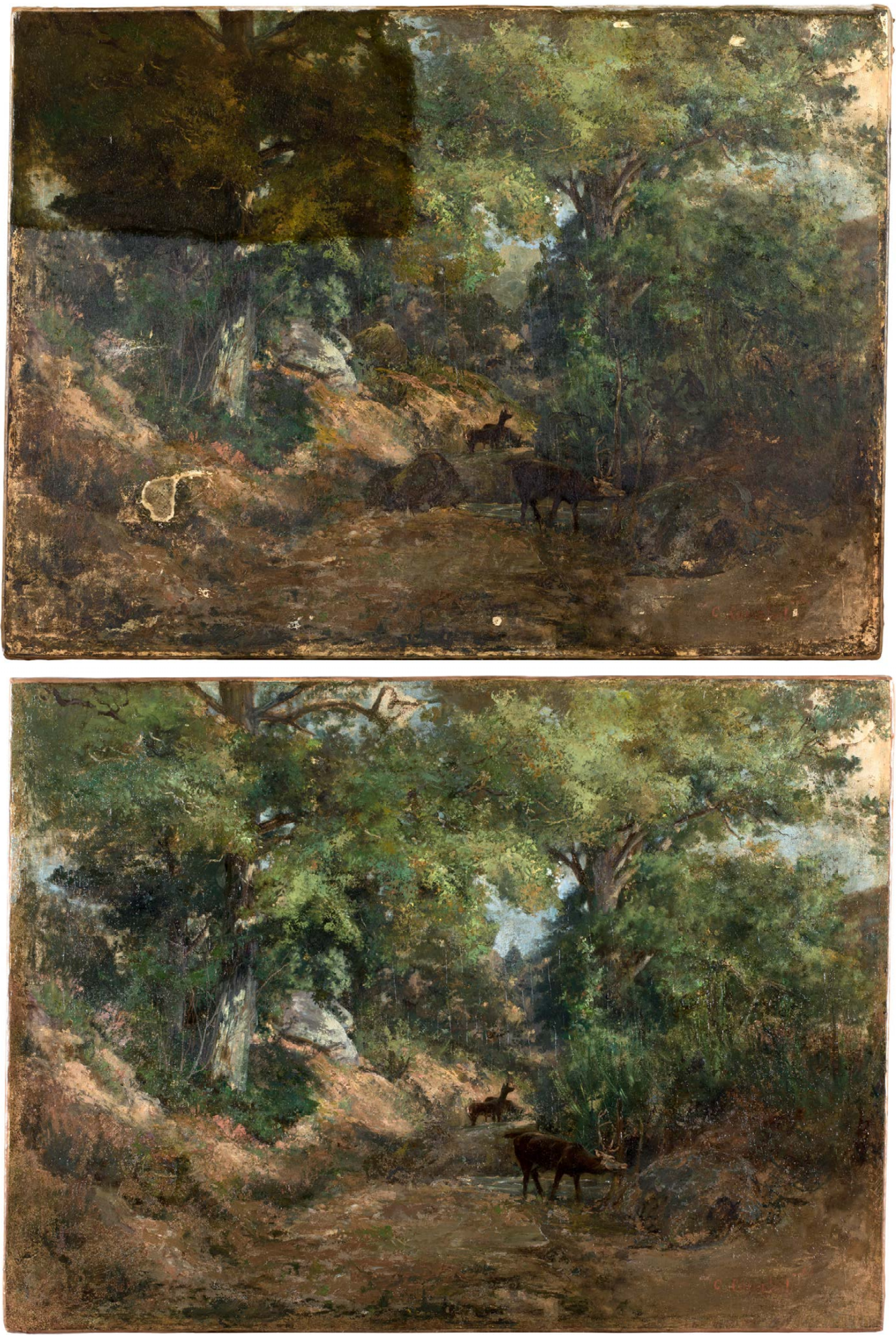

Fig. 4. Gustave Courbet, Le Cerf dans la forêt, 1867, huile sur toile ( $75 \times 100 \mathrm{~cm})$, musée du château de Flers, vue d'ensemble, intervention en cours (nettoyage). () C2RMF/T. Clot.

Fig. 5. Gustave Courbet, Le Cerf dans la forêt, 1867, huile sur toile $(75 \times 100 \mathrm{~cm})$, musée du château de Flers, vue d'ensemble après restauration. $\odot$ C2RMF/T. Clot. 
présentaient l'inconvénient du rinçage des résidus, quant aux gels rigides, leur mise en œuvre avec ce type de solvant n'était pas envisageable. L'utilisation de compresses, qui permettent d'exposer la surface du vernis avec plus d'efficacité, sans présenter l'inconvénient du rinçage des résidus, était ainsi la meilleure solution. Le protocole suivant a donc été mis au point : application du mélange de solvant au pinceau sur une compresse composée de plusieurs couches de papier absorbant ; pose d'un film hermétique non adhérent (Melinex ${ }^{\circledR}$ ) pour maintenir les solvants en contact sans évaporation par la face pendant 90 secondes (après ce délai, le vernis était partiellement imprégné dans les feuilles absorbantes), rinçage immédiat de la partie non solubilisée au bâtonnet (3 fois) avec le même mélange de solvant, puis avec des mélanges à la force de solubilité décroissante (éthanol $30 \%$-White Spirit $70 \%$ ).

Cette méthode a permis de supprimer le vernis de façon très homogène en limitant les frottements sur la couche picturale. Les résidus de repeints plus hétérogènes et parfois difficiles à solubiliser ont été supprimés de façon mécanique, sans compresses, le risque de ramollissement de la couche picturale originale étant possible.

Une partie des repeints a été conservée sur les parties les plus usées de l'œuvre, leur suppression représentant un risque de solubilisation des sous-couches, et demandant in fine un temps important dédié à la retouche de ces zones, ce qui aurait représenté un dépassement de budget considérable $^{8}$. Ces repeints ne gênent néanmoins pas la présentation de l'œuvre dans les parties où ils sont conservés, les zones sous-jacentes étant alors lacunaires.
Un vernis intermédiaire a été posé après vernissage, puis l'œuvre a été retouchée dans les parties lacunaires, notamment sur les bords, avec des matériaux de la gamme Gamblin Conservation Colors ${ }^{\circledR}$ (résine urée-aldéhyde Laropal ${ }^{\circledR}$ ).

\section{Conclusion}

Cette dérestauration a rendu au tableau une lisibilité de la surface picturale digne d'une œuvre de Courbet. Grâce à l'analyse fine de la radiographie, l'ampleur de l'intervention précédente a pu être évaluée pour prévoir sa dérestauration, mais la suppression d'un type de vernis peu courant sur une œuvre de cette qualité a demandé une mise en œuvre particulière. Grâce à la méthode de nettoyage par compresses, nous avons pu limiter considérablement les frottements qu'auraient induits un nettoyage au bâtonnet traditionnel ou le rinçage des résidus de gel de solvants. Cette technique révèle tout son intérêt pour des couches picturales fragiles et altérées comme dans le cas présenté ici. Le musée de Flers peut aujourd'hui présenter cette œuvre de Courbet, stabilisée par sa restauration, et réhabilitée par sa dérestauration.
Notes

1. Exp. Flers, 1997.

2. Toile de $30: 91,8 \times 72,9 \mathrm{~cm}$ dans les catalogues Lefranc \& Cie en 1863 et 1876 , $92 \times 73 \mathrm{~cm}$ dans le catalogue Lefranc de 1883 (Labreuche, 2011)

3. Le cadre a également fait l'objet d'une restauration, réalisée par l'atelier Sébastien David (Vernou-sur-Brenne). Il s'agit d'un cadre en bois doré avec ornements en pâte anglaise (Perle sur marie louise, cannelure et feuille d'acanthes dans les angles, et lauriers et cote d'angles) datable de 1860/1880.

4. Les interventions de restauration ont été conduites entre 2014 et 2016 avec la collaboration de plusieurs étudiantes en restauration : Marie Dion (université François Rabelais de Tours, stage de pratique de restauration et d'observation, participation au refixage), Pauline Fossier (CRBC Paris I, stage post-diplôme de pratique de restauration, participation au nettoyage), Nuria Jutglar Alvaro (ESCRBC de Catalunya, stage Erasmus-plus postdiplôme de pratique, participation au nettoyage), et Elisa Paolini (université de Florence, stage Erasmus-plus post-diplôme de pratique, participation au masticage). Elle a donné lieu à une exposition-dossier à la réouverture du musée en juin 2016, ainsi qu'à une conférence de présentation.

5. Avec un test de Feller et Cremonesi : chaque solvant et mélange de solvant possède une coordonnée en fonction des forces d'interactions intermodélulaires exprimées, Fd, Fp, Fh, modélisée dans un triangle (Triangle de Teas) - (Cremonesi, 2004). 6 . Polymère acrylique (résine en solution) iPBMA, Dégusa.

7. Cremonesi, 2004.

8. Les contraintes budgétaires, particulièrement pour les musées de petites collectivités, peuvent avoir un impact réel sur les choix de restauration.

\section{Bibliographie}

Courthion P., 1987, Tout l'œuvre peint de Courbet, Flammarion-Classique de l'Art, Paris.

Cremonesi P., 2004, L'Uso dei Solventi Organici nella Pulitura di Opere Policrome, Editore Il Prato, seconde édition.

Fernier R., 1978, La vie et l'œuvre de Gustave Courbet : catalogue raisonné, 2 vol., Fondation Wildenstein, Genève.

Flers, 1997, Julien Salles : 1829-1915, cat. exp., sous la direction de Chesneau-Dupin L. et Mulot F., Flers, musée du Château, 1997.

Labreuche P., 2011, Paris, capitale de la toile à peindre. XVIII ${ }^{e}-X I X^{e}$ siècle, CTHS-INHA, Paris, p. 300-301. 